\title{
O EXERCÍCIO PROFISSIONAL DO ENFERMEIRO ONCOLÓGICO NO NÍVEL TERCIÁRIO DE ATENÇÃo À SAÚDE
}

The oncology nurse professional practice at the tertiary level of health care

La práctica profesional de las enfermeras de oncología en la atención terciaria de salud

\section{Lívia Gomes da Silva1}

Resumo: O conhecimento geral e específico do enfermeiro oncológico é um subsídio para o planejamento assistencial em câncer. Este envolve a organização, sistematização de atividades e a tomada de decisão. Esse estudo discute as atividades privativas ao enfermeiro oncológico no processo de trabalho de enfermagem em uma unidade de internação em oncologia. Trata-se de uma revisão integrativa no portal CAPES e portal BIREME nos últimos 6 anos. Foram encontradas 36 atividades desenvolvidas pelos enfermeiros oncológicos, sendo que, destas, 58\% não são privativas ao referido profissional e $39 \%$ não são exclusivas do processo de enfermagem. É importante que o profissional enfermeiro conheça suas atividades privativas para que possa planejar de forma adequada e sistemática a assistência dos cuidados a serem prestados, facilite $o$ desenvolvimento do processo de trabalho da enfermagem e também o envolvimento da equipe multiprofissional em todo processo assistencial a ser prestado no ambiente hospitalar.

\section{Palavras-chave: Enfermagem oncológica; Hospitalização; Oncologia.}

Abstract: The general and specific knowledge nurses knowledge allowances to the appropriate care planning in cancer. It involves the organization, systematization of activities, and decision-making one. This study discusses the private oncological nurses in nursing work process in an oncology inpatient unit. This research its is integrative review on CAPES and BIREME portal in the last six years. There were 36 activities

\footnotetext{
${ }^{1}$ Universidade Federal do Rio de Janeiro - Mestre em Enfermagem/ UFRJ; Instituto Nacional do Câncer - Enfermeira Oncológica.
}

developed by oncology nurses, and of these $58 \%$ are not private oncologic nurses and $39 \%$ are not exclusive of the nursing process. It is important that the nurse know their private activities in order to plan properly and systematically the assistance of care, facilitate the development of the nursing work process and also the involvement of multidisciplinary team throughout the care process to be provided in the hospital.

\section{Key words: Oncology Nursing; Hospitalization; Medical Oncology.}

\section{Introdução}

O conhecimento pode ter várias definições, mas partindo de um conceito com base filosófica, define-se a mesmo como aquisição intelectual de um determinado campo do saber dominando e utilizando-o (1). O conhecimento é de suma importância na prática de enfermagem, pois segundo Vilma de Carvalho (2), é a partir do mesmo que se adquire um domínio do saber profissional.

Pode-se dizer que o enfermeiro oncológico possui, além dos conhecimentos gerais, inerentes a profissão, conhecimentos específicos acerca de oncologia. A Enfermagem Oncológica é uma especialidade reconhecida pelo Conselho Federal de Enfermagem (3). Para Rosa (4), o papel do enfermeiro oncológico envolve diversos procedimentos com vários graus de complexidade. A Lei do Exercício Profissional de Enfermagem (5) destaca que é privativo dos enfermeiros os cuidados de maior complexidade técnica e que exijam conhecimentos científicos e capacidade para tomada de decisão imediata. Portanto o profissional enfermeiro é fundamental para a 
assistência oncológica com qualidade e segurança.

Pode-se conceituar o especialista de enfermagem como:

[...] O profissional de alta proficiência que atingiu nível de elevada habilitação compatível com perícia, assessoria e consultoria, estimadas em graus sempre mais elevados de reconhecimento, na internalidade da profissão e fora dela [...]. (2)

A Ordem dos Enfermeiros de Portugal (6) faz uma consideração de extrema relevância no contexto do estudo em questão, ela destaca que a um enfermeiro especialista, além de suas responsabilidades e deveres comuns, compete também a melhoria contínua da qualidade dos cuidados prestados.

A oncologia é uma especialidade cheia de desafios e requer capacitação contínua dos profissionais, sendo uma área de densidade tecnológica e dinâmica (7). A presença de enfermeiros especialistas é extremamente necessária nas unidades que atendem pacientes oncológicos, pois além de possuírem conhecimento técnico-científico, estes possuem também responsabilidade $\mathrm{e}$ prática clínica capaz de dar subsídio ao planejamento assistencial e coordenação da equipe.

É interessante que as instituições de saúde atraiam e retenham profissionais qualificados em oncologia, visto que esses agregam

Mediante a isso, partiu-se para uma pesquisa teórica cujo objetivo foi discutir as atividades privativas ao enfermeiro oncológico, presentes no processo de trabalho de enfermagem em uma unidade de internação de oncologia.

\section{Referencial Teórico}

Acredita-se que o setor oncológico seja um ambiente cheio de fatores desencadeantes de estresse e diversas outras alterações emocionais para os profissionais que ali atuam (9). Este é um local de múltiplas conhecimentos acerca do câncer e suas implicações, modalidades terapêuticas e emergências oncológicas, dentre outros (8). Além disso, o enfermeiro qualificado em oncologia é capaz de planejar uma assistência voltada às reais necessidades de pacientes oncológicos e também apresentar tomada de decisão imediata diante da piora clínica dos mesmos e/ou outros problemas inerentes a essa área assistencial.

O exercício da enfermagem é normatizado e fiscalizado pelo Conselho Federal de Enfermagem (COFEN), que zela pela qualidade dos serviços prestados e pelo cumprimento da Lei do Exercício Profissional da Enfermagem (5) e Decreto $n^{\circ} 94.406$ (1987).

A partir das leis, resoluções e portarias do COFEN e Conselhos Regionais de Enfermagem (COREN), além de documentos do Ministério da Saúde e Agência Nacional de Vigilância Sanitária, será discutido o caráter privativo das atividades a serem desenvolvidas no âmbito hospitalar pelos enfermeiros oncológicos.

Conhecer as atividades a serem desenvolvidas pode facilitar o planejamento assistencial do enfermeiro oncológico, o desenvolvimento do seu processo de trabalho, o envolvimento da equipe de enfermagem, bem como da equipe multiprofissional. Com isso, promove-se uma maior qualidade e segurança na assistência a ser prestada no âmbito hospitalar.

demandas de baixa, média e alta complexidade, rotina acelerada e necessidade de tomada de decisão imediata, com a maior segurança e qualidade possível.

A identificação das intervenções mais utilizados em grupos específicos de pacientes, permite estabelecer os recursos necessários para execução da assistência, nível de cuidado, categoria do profissional envolvido e o tempo transcorrido para sua execução (10). Para a enfermagem oncológica, o conhecimento das principais atividades a serem realizadas com os pacientes e família, no âmbito hospitalar, pode facilitar a elaboração de um planejamento assistencial adequado às reais necessidades do indivíduo 
inserido no processo saúde-doença, à realidade da instituição de saúde, bem como aos outros profissionais de saúde envolvidos nesse processo assistencial.

O planejamento assistencial envolve a organização, priorização e a sistematização de atividades (11). Para que o trabalho seja devidamente organizado e sistematizado, parte-se, primeiramente, para a análise da situação e depois para o processo de tomada de decisão, considerando o que será feito, o objetivo de ser feito, se é prioritário ou não, quem deve fazer e como deve fazer. A primeira etapa do planejamento do trabalho do enfermeiro oncológico é identificar ou conhecer quais as atividades devem ser realizadas, quais recursos são necessários e quem deve realizá-las.

De acordo com a Regulamentação do Exercício Profissional de Enfermagem, a profissão é exercida privativamente pelo Enfermeiro, Técnico de Enfermagem e Auxiliar de Enfermagem. Cada uma destas possui atividades determinadas, sendo que algumas cabem, privativamente, ao enfermeiro e outras que não podem ser executadas pelos auxiliares de enfermagem, como ações assistenciais de enfermagem e participação na programação da assistência de enfermagem (5).

A assistência hospitalar em oncologia, dentre outras, exige ações assistenciais de enfermagem e não apenas ações de tratamento simples, reconhecimento e descrição de sinais vitais e cuidados de higiene e conforto. São necessários cuidados de enfermagem específicos, pautados numa programação assistencial previamente elaborada. Em oncologia, os cuidados de enfermagem permeiam peculiaridades relacionadas a doença, possível necessidade de procedimentos invasivos ou dolorosos e situações de gravidade e morte, tais fatos acabam demonstrando a complexidade na assistência de enfermagem ao paciente oncológico (11).

$\mathrm{O}$ adequado planejamento assistencial exige o envolvimento de toda equipe de enfermagem, ele é capaz de nortear os cuidados, de forma integral, a serem executados pela enfermeira e toda sua equipe. Devido a amplitude de ações a serem implementadas junto ao paciente oncológico na atenção terciária, é essencial que se caracterize a quais profissionais de enfermagem que cabem a execução das mesmas. Quando se identifica e divide as atribuições do cuidado a ser prestado por toda equipe é possível que toda equipe trabalhe de forma integrada, dedicando seus conhecimentos e habilidades específicas na assistência de enfermagem de acordo com a legislação do COFEN relacionada ao exercício profissional (5).

Quando o enfermeiro envolve toda equipe de enfermagem na assistência de forma organizada, ele facilita seu trabalho privativo de supervisão e monitorização das atividades realizadas por outros profissionais de enfermagem e verifica, também, a efetividade do processo de trabalho da equipe (11).

É importantíssimo caracterizar as atividades a serem realizadas pela equipe de enfermagem na assistência oncológica, considerando aqui o enfermeiro e o técnico de enfermagem. Essa caracterização pode ser um dos subsídios para o adequado planejamento assistencial, melhor otimização dos conhecimentos, habilidades, competência técnica e científica de ambas categorias e, consequentemente, facilitar a distribuições de tarefas de forma facilitada e integradora.

\section{Metodologia}

Para identificação das atividades do enfermeiro oncológico em uma unidade de internação de oncologia, foi realizada uma revisão integrativa no portal CAPES e portal BIREME, utilizando as palavras-chave "Enfermagem Oncológica" AND "Hospitalização".

O critério de tempo utilizado foi publicações dos últimos 08 anos, ou seja, publicações de 2010 a 2018, considerando o ano corrente, sendo encontrados 07 artigos em português no Portal BIREME e 11 artigos na CAPES, também em português, portanto um subtotal de 18 artigos. Ao verificar se os mesmos 
estavam repetidos, verificou-se que 03 artigos estavam repetidos nessa primeira análise.

Destaca-se que foi utilizado o critério de idioma Português a fim de identificar as atividades do enfermeiro oncológico realizadas no âmbito profissional no Brasil, pois as considerações e análises serão feitas a partir da normas, resoluções e leis do COFEN, que é órgão que regula e fiscaliza a profissão no país.

A partir da leitura dos resumos dos artigos e seleção dos mesmos para leitura do texto completo apenas daqueles que considerassem a assistência de enfermagem na oncologia em pacientes adultos, visto ser o público considerado no estudo em questão, foram selecionados 06 artigos. Logo após, iniciou-se a organização e tabulação dos dados e, para isso, foi elaborado um instrumento contendo os seguintes campos: título, revista/periódico, ano de publicação, abordagem e natureza do estudo, referência teórica, método coleta e análise de dados e atividades de enfermagem citadas ao longo da publicação.

Nos tais artigos foram identificadas as atividades realizadas pelo enfermeiro oncológico dentro do seu processo de trabalho assistencial no âmbito hospitalar e a partir daí realizada uma listagem pontuando cada uma delas.

Foi realizada uma análise dessas publicações selecionadas e, com base nos campos do roteiro supracitado, realizada uma síntese dos dados extraídos de forma descritiva a fim de identificar, contar e descrever as atividades realizadas durante o exercício profissional da equipe de enfermagem, durante a internação de pacientes oncológicos. A partir daí, foi reunido todo conhecimento já produzido sobre a temática no cenário brasileiro nos últimos 8 anos.

Por fim, foi realizada uma correlação da síntese elaborada com os conhecimentos produzidos com os documentos oficiais do Conselho Federal de Enfermagem, e foi identificado se tais atividades são privativas dos enfermeiros e quais categorias profissionais estão envolvidas nessa listagem.

\section{Resultados E Discussão}

$\mathrm{Na}$ busca bibliográfica, citada acima, para identificação das atividades dos enfermeiros, encontraram-se estudos com enfoques temáticos abrangendo a caracterização de pacientes, processo de hospitalização, assistência de enfermagem e análise de demandas de cuidados de enfermagem. Os aspectos metodológicos, cenário de estudo, população de estudo, ano de publicação e base de dados encontram-se listados no Quadro 1.

Quadro 1- Enfoques temáticos referentes aos artigos sobre Enfermagem oncológica e Hospitalização, encontrados no portal BIREME e CAPES, 2010-2018

\begin{tabular}{|l|l|l|l|l|l|}
\hline Enfoque temático & \multicolumn{1}{|c|}{$\begin{array}{c}\text { Aspectos } \\
\text { metodológicos }\end{array}$} & $\begin{array}{l}\text { Cenário do } \\
\text { estudo }\end{array}$ & $\begin{array}{c}\text { População } \\
\text { do estudo }\end{array}$ & Ano & Base de dados \\
\hline $\begin{array}{l}\text { Caracterização de } \\
\text { pacientes; processo de } \\
\text { hospitalização; } \\
\text { assistência de } \\
\text { enfermagem }\end{array}$ & $\begin{array}{l}\text { Qualitativa: } \\
\text { entrevista }\end{array}$ & $\begin{array}{l}\text { Hospital } \\
\text { Universitário }\end{array}$ & $\begin{array}{l}\text { Pacientes; } \\
\text { equipe de } \\
\text { enfermagem }\end{array}$ & 2012 & $\begin{array}{l}\text { Portal BIREME } \\
\text { e base de dados } \\
\text { BDENF }\end{array}$ \\
\hline $\begin{array}{l}\text { Assistência de } \\
\text { enfermagem a partir } \\
\text { da vivência da equipe }\end{array}$ & $\begin{array}{l}\text { Qualitativa: } \\
\text { entrevista }\end{array}$ & $\begin{array}{l}\text { Hospital } \\
\text { Universitário }\end{array}$ & $\begin{array}{l}\text { Equipe de } \\
\text { enfermagem }\end{array}$ & 2011 & $\begin{array}{l}\text { Portal } \\
\text { BIREME e } \\
\text { base de dados } \\
\text { BDENF }\end{array}$ \\
\hline $\begin{array}{l}\text { Assistência de } \\
\text { enfermagem; análise }\end{array}$ & $\begin{array}{l}\text { Qualitativa: } \\
\text { estudo de caso }\end{array}$ & $\begin{array}{l}\text { Hospital } \\
\text { Universitário }\end{array}$ & Enfermeiros & 2017 & $\begin{array}{l}\text { Portal } \\
\text { BIREME }\end{array}$ \\
\hline
\end{tabular}




\begin{tabular}{|l|l|l|l|l|l|}
\hline $\begin{array}{l}\text { das demandas de } \\
\text { cuidados de } \\
\text { enfermagem }\end{array}$ & & & & & $\begin{array}{l}\text { base de dados } \\
\text { BDENF }\end{array}$ \\
\hline $\begin{array}{l}\text { Assistência de } \\
\text { enfermagem }\end{array}$ & $\begin{array}{l}\text { Qualitativa: grupo } \\
\text { focal }\end{array}$ & $\begin{array}{l}\text { Hospital } \\
\text { oncológico }\end{array}$ & Enfermeiros & 2010 & CAPES \\
\hline $\begin{array}{l}\text { Assistência de } \\
\text { enfermagem }\end{array}$ & $\begin{array}{l}\text { Quantitativa: } \\
\text { análise } \\
\text { documental }\end{array}$ & $\begin{array}{l}\text { Hospital } \\
\text { Universitário }\end{array}$ & Pacientes & 2013 & CAPES \\
\hline $\begin{array}{l}\text { Processo de } \\
\text { hospitalização }\end{array}$ & $\begin{array}{l}\text { Qualitativa: } \\
\text { entrevista }\end{array}$ & $\begin{array}{l}\text { Hospital } \\
\text { Geral }\end{array}$ & Cuidadores & 2012 & CAPES \\
\hline
\end{tabular}

No processo de trabalho da enfermagem existem tanto atividades privativas aos enfermeiros, quanto àquelas que podem ser realizadas por toda equipe de enfermagem e, também, outras que podem ser realizadas pela equipe multiprofissional no ambiente hospitalar.

Segundo a lei $n^{\circ} 7498$ de 1986 do conselho federal de enfermagem, que regulamenta o exercício profissional da enfermagem (5), o enfermeiro exerce todas as atividades de enfermagem, sendo privativo a esse profissional o planejamento e coordenação dos serviços de enfermagem, prescrição da assistência de enfermagem, cuidados diretos de enfermagem a pacientes graves com risco de vida, cuidados de enfermagem de maior complexidade técnica e que exijam conhecimentos de base científica e capacidade de tomar decisões imediatas, participação no planejamento, elaboração dos planos assistenciais de saúde e prevenção e controle sistemático de danos que possam ser causados à clientela durante a assistência.

No quadro 2, está apresentada a lista das atividades desenvolvidas dentro do processo de trabalho da enfermagem oncológica em unidades de internação, de acordo com os resultados da revisão bibliográfica supracitada, e suas respectivas categorias no que tange ao processo de enfermagem, caráter privativo e categorias profissionais envolvidas em cada atividade.

\begin{tabular}{|c|c|c|c|}
\hline Atividades & $\begin{array}{c}\text { Atividades } \\
\text { exclusivas do } \\
\text { processo de } \\
\text { enfermagem }\end{array}$ & $\begin{array}{l}\text { Categoria profissional } \\
\text { também envolvida nessa } \\
\text { Atividade }\end{array}$ & É privativa? \\
\hline Administrar medicações & SIM & $\begin{array}{l}\text { Enfermeira; Técnico de } \\
\text { enfermagem. }\end{array}$ & NÃO \\
\hline $\begin{array}{l}\text { Administrar sangue e } \\
\text { hemoderivados }\end{array}$ & SIM & Enfermeira & SIM \\
\hline Aferir sinais vitais & SIM & $\begin{array}{l}\text { Enfermeira; Técnicos de } \\
\text { enfermagem. }\end{array}$ & NÃO \\
\hline $\begin{array}{l}\text { Auxiliar médicos em } \\
\text { procedimentos }\end{array}$ & $\mathrm{NÃO}$ & $\begin{array}{l}\text { Enfermeira; Técnicos de } \\
\text { enfermagem; Médicos. }\end{array}$ & $\mathrm{NÃO}$ \\
\hline $\begin{array}{l}\text { Avaliar grau de dependência de } \\
\text { cuidados de enfermagem }\end{array}$ & SIM & Enfermeira & SIM \\
\hline $\begin{array}{l}\text { Avaliar presença de familiares, } \\
\text { acompanhantes e visitas }\end{array}$ & $\mathrm{NÃO}$ & $\begin{array}{l}\text { Enfermeira; Técnicos de } \\
\text { enfermagem; Serviço. } \\
\text { Social; Psicologia }\end{array}$ & NÃO \\
\hline Capacitar equipe de enfermagem & SIM & Enfermeira & SIM \\
\hline
\end{tabular}




\begin{tabular}{|l|l|l|l|}
\hline Coletar sangue e hemocultura & NÃO & Enfermeira & SIM \\
\hline Cuidados com cateteres e punções & SIM & Enfermeira & SIM \\
\hline Delegar cuidados necessários & SIM & Enfermeira & SIM \\
\hline $\begin{array}{l}\text { Detectar necessidades de acordo } \\
\text { com modalidade terapêutica ou } \\
\text { cuidado prestado }\end{array}$ & NÃO & Enfermeira; Médicos & NÃO \\
\hline $\begin{array}{l}\text { Diminuir índice de infecção } \\
\text { hospitalar }\end{array}$ & NÃO & $\begin{array}{l}\text { Enfermeira; Técnicos de } \\
\text { enfermagem. }\end{array}$ & NÃO \\
\hline $\begin{array}{l}\text { Discutir com administração sobre } \\
\text { estrutura física ideal para paciente } \\
\text { e família no hospital }\end{array}$ & NÃO & Enfermeira; Administrador & NÃO \\
\hline $\begin{array}{l}\text { Elaborar plano de cuidados } \\
\text { diariamente }\end{array}$ & SIM & Enfermeira & SIM \\
\hline $\begin{array}{l}\text { Encaminhar e receber } \\
\text { medicamentos e quimioterapia }\end{array}$ & SIM & $\begin{array}{l}\text { Enfermeira } \\
\text { Técnicos de enfermagem; }\end{array}$ & NÃO \\
\hline Gerenciar cuidado & SIM & Enfermeira & SIM \\
\hline $\begin{array}{l}\text { Identificar e implementar ações } \\
\text { para necessidades dos pacientes }\end{array}$ & SIM & Snfermeira \\
\hline
\end{tabular}

Quadro 2- Descrição de atividades desenvolvidas no processo de trabalho da enfermagem em unidade de internação de oncologia com correlação dos profissionais envolvidos, critérios de exclusividade no processo de enfermagem e se privativo ou não ao enfermeiro oncológico.

(continuação)

\begin{tabular}{|c|c|c|c|}
\hline Atividades & $\begin{array}{c}\text { Atividades } \\
\text { exclusivas do } \\
\text { processo de } \\
\text { enfermagem } \\
\end{array}$ & $\begin{array}{l}\text { Categoria profissional } \\
\text { também envolvida nessa } \\
\text { atividade }\end{array}$ & $\begin{array}{l}\text { É } \\
\text { privativa } \\
?\end{array}$ \\
\hline Manipulação de medicamentos & SIM & $\begin{array}{l}\text { Enfermeira; Técnicos de } \\
\text { enfermagem }\end{array}$ & $\mathrm{NÃO}$ \\
\hline Marcar exames & $\mathrm{NÃO}$ & Administrativos & $\mathrm{NÃO}$ \\
\hline $\begin{array}{l}\text { Orientar paciente e família } \\
\text { quanto a rotinas hospitalares }\end{array}$ & NÃO & $\begin{array}{l}\text { Enfermeira; } \\
\text { Técnicos de enfermagem; } \\
\text { Serviço social }\end{array}$ & $\mathrm{NÃO}$ \\
\hline $\begin{array}{l}\text { Orientar paciente/família } \\
\text { quanto a autocuidado na alta }\end{array}$ & SIM & $\begin{array}{l}\text { Enfermeira; } \\
\text { Técnicos de enfermagem }\end{array}$ & $\mathrm{NÃO}$ \\
\hline $\begin{array}{l}\text { Planejamento e organização do } \\
\text { cuidado }\end{array}$ & SIM & Enfermeira & SIM \\
\hline $\begin{array}{l}\text { Prever e prover condições de } \\
\text { segurança e conforto do } \\
\text { paciente e família }\end{array}$ & $\mathrm{NÃO}$ & $\begin{array}{l}\text { Enfermeira; Técnicos de } \\
\text { enfermagem; } \\
\text { Administrador }\end{array}$ & $\mathrm{NÃO}$ \\
\hline $\begin{array}{l}\text { Prever e prover recursos } \\
\text { materiais ou suprimentos }\end{array}$ & $\mathrm{NÃO}$ & $\begin{array}{l}\text { Enfermeira; Farmacêutico; } \\
\text { Médicos; Administrador }\end{array}$ & SIM \\
\hline Promover apoio nutricional & $\mathrm{NÃO}$ & $\begin{array}{l}\text { Nutricionista; Enfermeira; } \\
\text { Técnicos de enfermagem }\end{array}$ & $\mathrm{NÃO}$ \\
\hline Promover apoio psicológico & $\mathrm{NÃO}$ & $\begin{array}{l}\text { Enfermeira; Técnicos de } \\
\text { enfermagem; Médicos; }\end{array}$ & $\mathrm{NÃO}$ \\
\hline
\end{tabular}




\begin{tabular}{|c|c|c|c|}
\hline & & Serviço social & \\
\hline Promover cuidado integral & SIM & Enfermeira & SIM \\
\hline Proteger direitos dos pacientes & NÃO & $\begin{array}{l}\text { Serviço social; } \\
\text { Enfermeira; Médicos; } \\
\text { Psicólogas; Técnicos de } \\
\text { enfermagem }\end{array}$ & NÃO \\
\hline $\begin{array}{l}\text { Prever/prover recursos } \\
\text { humanos de enfermagem }\end{array}$ & SIM & Enfermeira & SIM \\
\hline Realizar cuidados na admissão & SIM & $\begin{array}{l}\text { Enfermeira; Técnicos de } \\
\text { enfermagem }\end{array}$ & NÃO \\
\hline Realizar curativos & SIM & $\begin{array}{l}\text { Enfermeira; Técnicos de } \\
\text { enfermagem }\end{array}$ & NÃO \\
\hline $\begin{array}{l}\text { Realizar medidas de higiene e } \\
\text { conforto }\end{array}$ & SIM & $\begin{array}{l}\text { Enfermeira; Técnicos de } \\
\text { enfermagem }\end{array}$ & NÃO \\
\hline Realizar mudança de decúbito & SIM & $\begin{array}{l}\text { Enfermeira; Técnicos de } \\
\text { enfermagem }\end{array}$ & NÃO \\
\hline $\begin{array}{l}\text { Realizar Sistematização da } \\
\text { Assistência de Enfermagem } \\
\text { (SAE) }\end{array}$ & SIM & Enfermeira & SIM \\
\hline $\begin{array}{l}\text { Realizar supervisão da } \\
\text { assistência prestada }\end{array}$ & SIM & Enfermeira & SIM \\
\hline $\begin{array}{l}\text { Realizar troca de informação } \\
\text { com equipe multiprofissional }\end{array}$ & $\mathrm{NÃO}$ & $\begin{array}{l}\text { Enfermeira; Serviço social; } \\
\text { Psicóloga; Médicos }\end{array}$ & NÃO \\
\hline
\end{tabular}

Na correlação dessas atividades com normas, resoluções e Lei do Exercício Profissional da enfermagem, verificou-se aquelas que eram privativas do enfermeiro e quais não eram (Quadro 2). O Gráfico 1 ilustra a porcentagem das atividades privativas e não privativas dentre a listagem presente acima.

Gráfico 1- Relação das atividades privativas e não privativas do enfermeiro oncológico em unidade de internação.

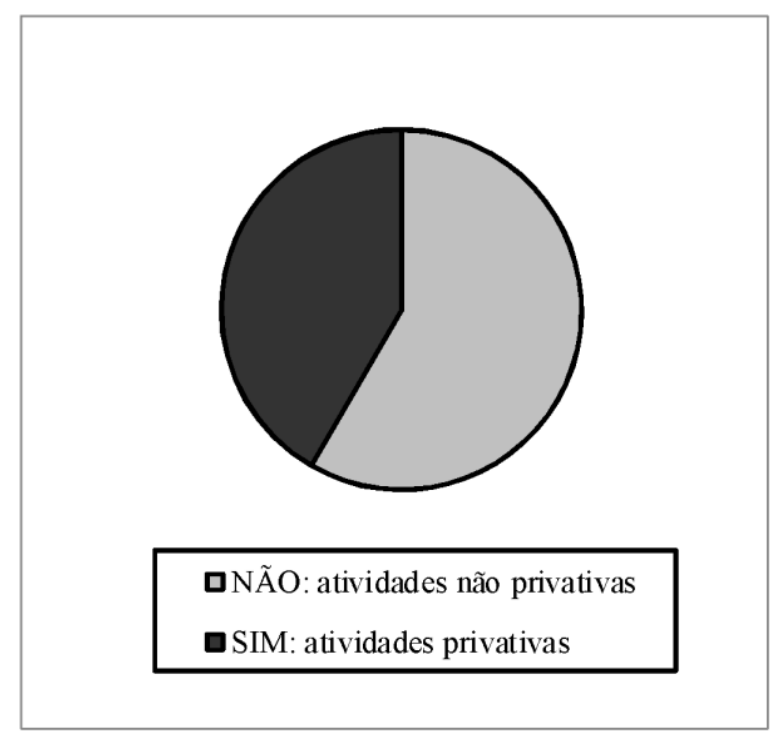


É responsabilidade do enfermeiro a coordenação da passagem de plantão, sendo esta uma ferramenta utilizada para assegurar o fluxo e proporcionar informações para a continuidade da assistência (12). Na listagem supracitada, três das atividades listadas se relacionam com a passagem de plantão: envolver equipe de enfermagem na SAE, identificar e implementar ações mediante necessidades dos pacientes e realizar troca de informação com equipe multiprofissional. Nessas atividades, as informações dos pacientes são repassadas aos profissionais que realizarão sua assistência durante a sua jornada de trabalho. A equipe será envolvida em todo o processo assistencial e as principais necessidades dos pacientes serão elencadas, bem como as ações implementadas durante a jornada de trabalho anterior.

Já a classificação de pacientes ou a avaliação do grau de dependência dos pacientes faz parte do planejamento e coordenação dos serviços de enfermagem, além do planejamento do plano assistencial de enfermagem, pois o sistema de classificação de pacientes pode ser considerado uma forma de categorização do paciente em relação à assistência de enfermagem (13), por meio desta, é possível estimar a carga de trabalho e prever o número adequado de recursos humanos de enfermagem para uma assistência de enfermagem de qualidade e segura. Duas atividades listadas acima se relacionam com essa classificação: classificação dos pacientes de acordo com seu grau de complexidade e divisão de escala diária de cuidados dos técnicos de enfermagem e enfermeiras plantonistas. Tais atividades também estão inclusas no artigo 11 da lei do exercício profissional da enfermagem (5), o qual destaca as atividades privativas do Enfermeiro.

Em relação a sistematização da assistência de enfermagem, considera-se, a partir da resolução 358 do Conselho Federal de Enfermagem, em 2009, que dispõe sobre a
SAE (14), que cabe ao enfermeiro a liderança na execução do processo de enfermagem e é privativo desse profissional o diagnóstico de enfermagem e a prescrição das ações ou intervenções a serem realizadas. Já com relação a previsão e provisão de recursos materiais e suprimentos na fase do aprazamento das prescrições médicas, destaca-se que o enfermeiro é o profissional que possui competência técnica científica para tal atividade, visto os conhecimentos adquiridos durante a graduação em enfermagem.

A partir da revisão integrativa realizada no presente estudo, se destacaram aqui um total de 36 atividades, $61,5 \%$ dessas estão inseridas, exclusivamente, no processo de enfermagem e $38,5 \%$ não são exclusivas do processo de enfermagem. As categorias profissionais envolvidas nas atividades destacadas são: técnicos de enfermagem, médicos, assistentes sociais, psicólogos, enfermeiros, administradores e pessoal do serviço administrativo.

Infere-se também que 59\% das atividades de enfermagem não são privativas dos enfermeiros.

Discutiu-se aqui as atividades de caráter privativo do enfermeiro. Para abordar as demais atividades que envolvem outras categorias profissionais, é importante conhecer as leis que regulamentam o exercício profissional dessas categorias e um aprofundamento maior na sua temática.

\section{Considerações Finais}

O conhecimento gera saber profissional (2) e aliado a esse, advém, também, mais responsabilidades. É conhecido por meio da Lei do Exercício Profissional (5) que o enfermeiro possui um número maior de atividades a serem desempenhadas na assistência de enfermagem, pois a ele 
compete tanto o cuidado direto quanto o cuidado indireto.

Conceituando o cuidado direto e indireto, verifica-se, segundo Christovan, Porto e Oliveira (15), que o processo de trabalho do enfermeiro envolve o cuidado direito, a assistência propriamente dita prestada às pessoas doentes e o cuidado indireto, as ações conhecidas como administrativas ou burocráticas.

É de suma importância que todos os membros da equipe multiprofissional saibam quais são suas atribuições profissionais e deveres dentro do processo assistencial. O enfermeiro deve conhecer suas atribuições e sua competência assistencial no âmbito hospitalar. Esse profissional, o enfermeiro, é responsável pelo gerenciamento do cuidado prestado, e no caso do estudo em questão, o enfermeiro oncológico, profissional capacitado para assistir pacientes que exigem assistência de média e alta complexidade, devido seu processo de saúde-doença oncológica, possui diversas responsabilidades e atribuições privativas, que se não forem realizadas com segurança e qualidade, podem prejudicar o processo de reabilitação da pessoa doente e até ocasionam sua piora clínica.

Há de se dizer que o profissional enfermeiro deve valorizar o que lhe compete privativamente, saber delegar com segurança e respaldo do seu órgão regulador profissional as atividades para o restante da equipe de enfermagem. Deve também envolver os demais profissionais da área de saúde responsáveis pela assistência prestada no âmbito hospitalar, para a coparticipação nas atividades cujas quais estão envolvidos e que não são privativas de um único profissional. Isso pode diminuir a sobrecarga do profissional enfermeiro, promover uma maior troca de conhecimento multiprofissional e, consequentemente, gerar uma maior qualidade na assistência prestada. 


\section{Referências}

1. Japiassú $H$, Marcondes D. Dicionário básico de Filosofia. 3 edição. Rio de Janeiro: Jorge Zahar Editor, 2001

2. Carvalho V. Para uma epistemologia da Enfermagem: tópicos de crítica e contribuição. Rio de Janeiro: UFRJ/EEAN, 2013.

3. Conselho Federal de Enfermagem. Resolução COFEN 389: Atualiza, no âmbito do Sistema Cofen/Conselhos Regionais de Enfermagem, os procedimentos para registro de título de pósgraduação lato e scrito sensu concedido a enfermeiros e lista as especialidades. RJ: Conselho Federal de Enfermagem; 2011.

4. Rosa L M. O cuidado de enfermagem no itinerário terapêutico da pessoa com diagnóstico de câncer. 2007. Dissertação (Mestrado) - Universidade Federal de Santa Catarina, Centro de Ciências da Saúde. Programa de Pós-Graduação em Enfermagem, Florianópolis, 2007

5. Conselho Federal de Enfermagem. Lei COFEN 7498: Dispõe sobre a regulamentação do exercício da Enfermagem e dá outras providências.RJ: Conselho Federal de Enfermagem; 1986.

6. Ordem dos Enfermeiros. Regulamento OE 123: Regulamento das competências comuns do Enfermeiro Especialista. Lisboa: Ordem dos Enfermeiros; 2011.

7. Hercos TM,Vieira FS, Oliveira MS, Buetto LS, Shimura CMN, et al. O trabalho dos profissionais de Enfermagem em Unidades de Terapia Intensiva na assistência ao paciente oncológico. Revista Brasileira de Cancerologia. 2014; 60 (1):51-58

\section{Participação dos autores}

Gomes, LS atou na concepção teórica, coleta de dados, analise estática e elaboração e redação final do texto.

Recebido: 24.08.2017

Revisado: 30.08.2017

Aprovado: 03.08.2018
8. Bordigon M, Monteiro MI, Mai S, Martins MFSV, Rech CRA, et al. Satisfação e insatisfação no trabalho de profissionais de enfermagem da oncologia do Brasil e Portugal. Rev Texto \& Contexto Enfermagem. 2015;24(4): 925-33.

9. Viana TS. O estresse da enfermagem no setor oncológico. Revista científica Onlinez, Maringá, 2010.

10. Bulechek GM, Dochterman MJ, Butcher H. Classificação das Intervenções de Enfermagem. 5edição. São Paulo: Elsevier, 2010.

11. Silva, RCV,Cruz EA. Planejamento da assistência de enfermagem em oncologia: estudo da estrutura das representações sociais de enfermeiras. Rev Gaúcha Enfermagem. 2014; 35 (1): 116123.

12. Fenili RM. A passagem de plantão: um elo de ligação no processo de trabalho da enfermagem. Revista Ciências da Saúde. 1996; 1(1):97-113

13. Vigna CP, Perroca MG. Utilização de sistema de classificação de pacientes e métodos de dimensionamento de pessoal de enfermagem. Arquivo Ciências Saúde. 2002 Jan; 14 (1):8-12.

14. Conselho Federal de Enfermagem. Resolução COFEN 358: Dispõe sobre a Sistematização da Assistência de Enfermagem e a implementação do Processo de Enfermagem em ambientes, públicos ou privados, em que ocorre o cuidado profissional de Enfermagem, e dá outras providências. RJ: Conselho Federal de Enfermagem; 2009.

15. Christovam BP, Porto IS, Oliveira DC. Gerência do cuidado de enfermagem em cenários hospitalares: a construção de um conceito. Rev Esc Enferm. 2012; 46(3):734-41. 